\title{
Antimicrobial photodynamic therapy and light-activated disinfection efficacy in decontaminating titanium implant surfaces - In vitro study
}

\author{
Bleron Azizi ${ }^{1,4}$, Veranda Azizi ${ }^{2}$, Marija Peeva-Petreska ${ }^{3}$, Marko Vuletic ${ }^{4}$, Mato \\ Susic $^{4}$, Dragana Gabric ${ }^{4}$
}

1) Faculty of Dentistry, AAB College, Prishtina, Kosovo

2) PhD Studies, School of Dental Medicine, University of Zagreb, Zagreb, Croatia

3) Department of Oral Surgery, Faculty of Dentistry, University Ss. Cyril and Methodius, Skopje, North Macedonia

4) Department of Oral Surgery, School of Dental Medicine, University of Zagreb, Zagreb, Croatia

\section{Background and Aim}

As dental implants are becoming a common dental treatment option, there is also an increase in the frequency of peri-implantitis. The main cause of periimplantitis are considered to be the microorganisms living on the implant surface. The goal of treating peri-implantitis is to stop the inflammatory process and the bone loss that occurred as a result of the disease. Antimicrobial photodynamic therapy (PDT) is a procedure in which a photosensitive dye is activated by light and leads to the generation of cytotoxic reactive oxygen species, predominantly singlet oxygen $(7,8)$. The aim of this in vitro study was to evaluate and compare the effect of antimicrobial photodynamic therapy and light-activated disinfection on contaminated titanium dental implants. In addition Scanning Electron

\section{Methods and Materials}

Materials and Methods: The study was conducted on 72 titanium dental implants (Bredent ${ }^{\circledR}$, Senden, Germany) contaminated with a bacterial suspension prepared from three different bacterial species (Prevotella intermedia, Aggreggatibacter actinomycetemcomitans and Porphyromonas gingivalis) and were incubated in anaerobic conditions for 72 hours. The implants were randomly divided into four experimental groups and two control groups ( $\mathrm{n}=12$ each), according to the following treatment protocols: Group 1 (PDT1): PDT with toluidine blue (Fig.1); Group 2 (PDT2): PDT with phenothiazine chloride dye (Fig.2); Group 3 (LAD): light emitting diode (LED) with toluidine blue; Group 4 (TB): treatment with only toluidine blue. In the positive control (PC) group, the implants were treated with a $0.2 \%$ chlorhexidine-based solution, and in the negative control (NC) group, no treatment was used. After treatment the implants were put into Eppendorf tubes containing PBS, they were vortexed and $0.1 \mathrm{ml}$ was taken out of each tube and placed in Brucella agar plates. After 72h colony forming units (CFUs) were counted.
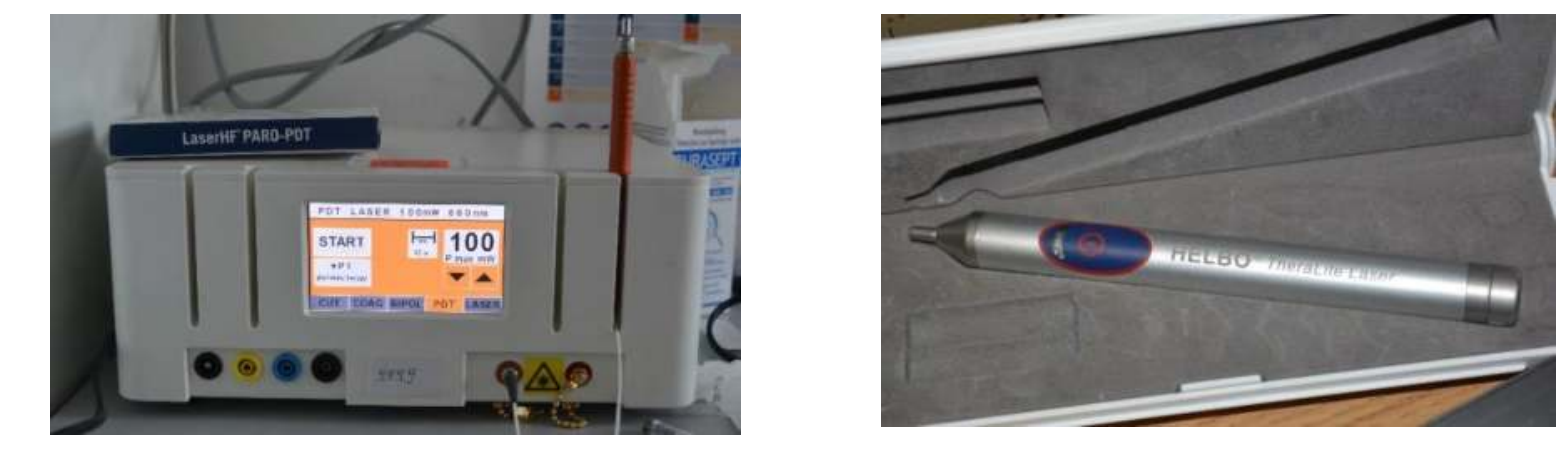

Figure 1. LaserHF® (Hager Werken, Duisburg, Germany) - PDT1. Figure 2. Helbo® (Helbo Photodynamic Systems GmbH \& Co KG, Grieskirchen Austria) - PDT2
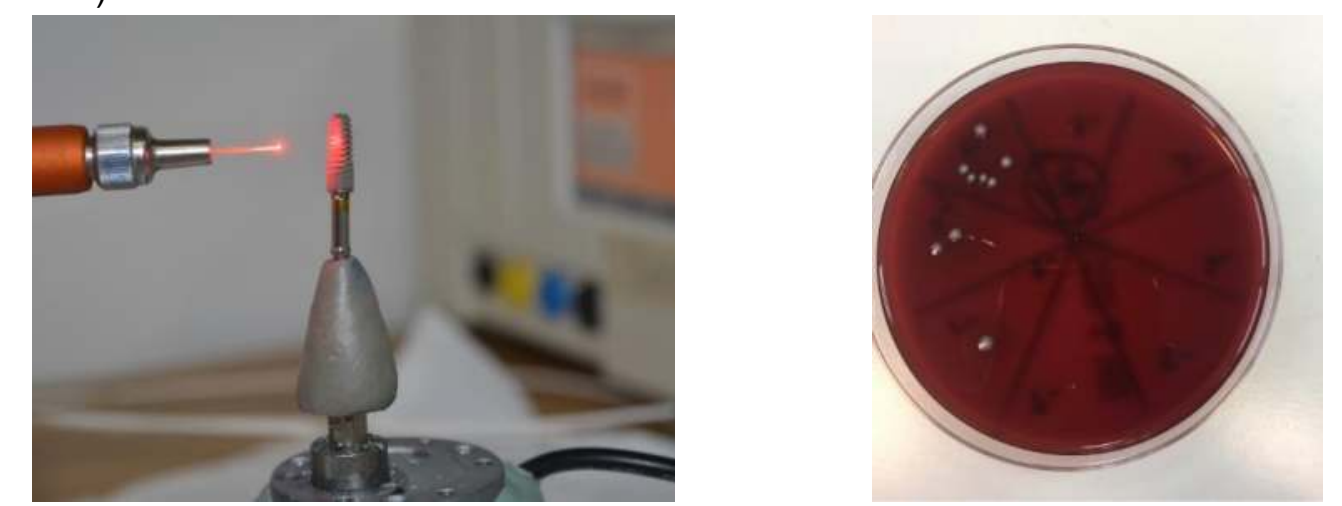

Figure 3. A contaminated titanium implant placed on a electric rotational motor ( $10 \mathrm{rpm}$ ) and treated for 60 seconds with PDT1.

Figure 4. Brucella agar plate with visible bacterial colonies.

\section{Results}

Results: The highest bacterial reduction was recorded in the PDT1 (98.3\%) and PDT2 $(\mathbf{9 7 . 8 \% )}$ groups both having statistically significant reduction compared to NC group $(<0.05)$. LAD was less effective than PDT1 and PDT2, without statistically significant difference compared with NC or any other treatment group. TB was the least effective treatment in terms of both the total bacterial count and the individual count for each bacterial species. There were no surface alterations after the use of PDT or LAD on the implant surfaces (Figure 5-8).
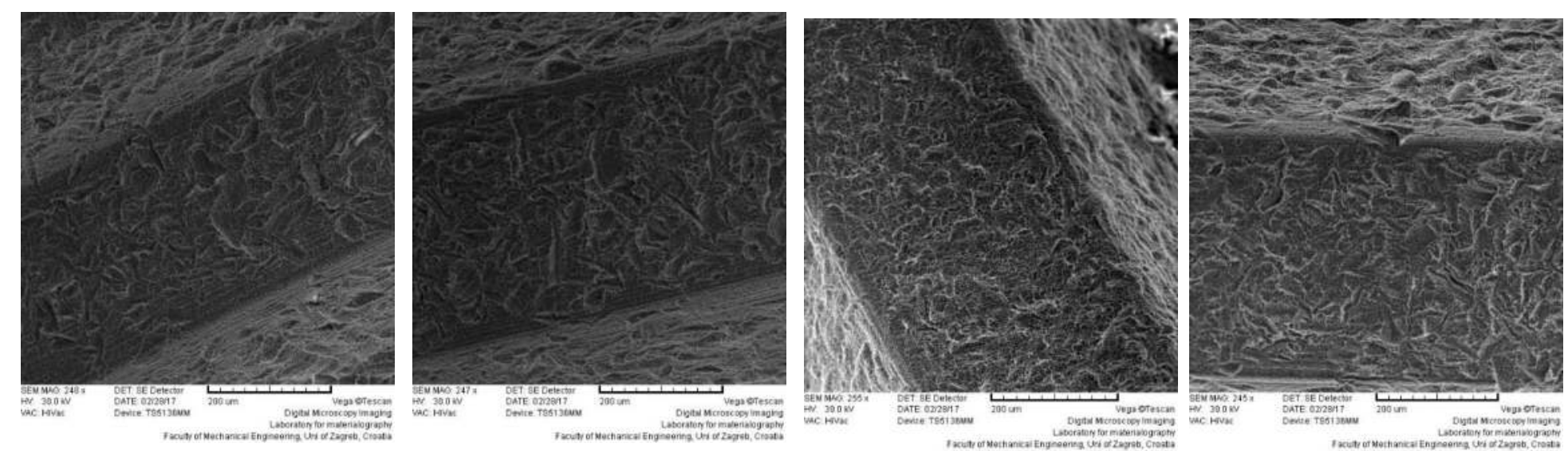

Figure 5. SEM image of an unopened sterile titanium implant.

Figure 6. SEM image of an implant treated with PDT1.

Figure 7. SEM image of an implant treated with PDT2.

Figure 8. SEM image of an implant treated with LAD.

\section{Conclusion}

Conclusion: Antimicrobial photodynamic therapy can be a successful alternative option for decontaminating titanium dental implants. An effectitve treatment protocol for decontaminating implant surfaces should be established and the potential use of PDT and LAD should be further investigated in clinical studies.

\section{References}

1.Mombelli A, Lang NP. The diagnosis and treatment of peri-implantitis. Periodontol 2000 1998;17:63-76.

2.Takasaki AA, Aoki A, Mizutani K, et al. Application of antimicrobial photodynamic therapy in periodontal and peri-implant diseases. Periodontol 2000 2009;51:109-140.

3. Dörtbudak O, Haas R, Bernhart T, Mailath-Pokorny G. Lethal photosensitization for decontamination of implant surfaces in the treatment of peri-implantitis. Clin Oral Implants Res 2001;12:104-108. 